\title{
Editorial
}

\section{Immunoadsorption Versus Therapeutic Plasma Exchange. Will Fibrinogen Make the Difference?}

\author{
Patrick M. Honoré Rita Jacobs Elisabeth De Waele Viola Van Gorp Herbert D. Spapen \\ ICU Department, Universitair Ziekenhuis Brussel, Vrije Universiteit Brussel, Brussels, Belgium
}

Therapeutic plasma exchange (TPE) already has a long service record in modern medicine. Throughout the years, TPE has steadily evolved from a centrifugationbased technique essentially used in bloodbanking procedures toward an easily applicable and efficient hemofiltration-steered modality in critically ill patients [1]. In support of the benefit of TPE was its mechanistic potential to remove injurious or noxious large molecular-weight substances (i.e., auto-antibodies, immune complexes, myeloma light chains, endotoxin, and cryoglobulins) and lipids such as cholesterol or triglycerides [2]. Subsequently, indications for TPE progressively also expanded encompassing thrombotic microangiopathies (e.g., Moscowitz syndrome), specific auto-immune diseases (e.g., Guillain-Barré, Goodpasture's, and anti-phospholipid syndrome), as well as rescue treatment of a wide variety of connective tissue and neurological disorders [3-7].

Classically, exchange is realized with human albumin to minimize potential allergic reactions induced by fresh frozen plasma (FFP) [8]. Proteins are removed from the plasma by an exchange process and by microthrombosis. In this scenario, levels of plasma constituents, including coagulation factors, at first substantially decrease and then gradually regain pre-treatment levels. However, the decrease and recovery rate of specific factors may vary. Fibrinogen levels, for instance, are markedly reduced and their return to baseline values may take days, even in patients with normal liver function [9]. Although a reduction of coagulation parameters in general does not necessarily imply more hemorrhagic complications [10], a low fibrinogen concentration is definitely associated with an increased bleeding risk [11]. This bleeding diathesis is also prolonged in TPEtreated patients with extremely low and/or slowly recovering fibrinogen levels. Importantly, such a scenario cannot be predicted, which precludes timely anticipation and prevention [12]. Over the years, strategies have been developed to tackle a low fibrinogen condition after TPE providing FFP targeting a fibrinogen level above $100 \mathrm{mg} /$ dl [13].

Unlike TPE, immunoadsorption (IAS) is a blood-purification technique that enables the selective removal of immunoglobulins (Ig) from separated plasma through high-affinity adsorbers. IAS is currently used for treatment of a large variety of antibody-mediated or immunological diseases (e.g., humoral transplant rejection, lupus nephritis, multiple sclerosis) [3, 14].

In this issue of Blood Purification, Zollner et al. retrospectively compared TPE, IAS and a TPE/IAS combination in $67 \mathrm{pa}-$ tients with a broad spectrum of immunoand neuropathies [15]. All methods reduced plasma fibrinogen levels by a fixed percentage independently of pre-treatment concentrations. However, fibrinogen levels fell below $100 \mathrm{mg} / \mathrm{dl}$ in one-fifth of the patients treated with either TPE or TPE/IAS whereas it rarely decreased after IAS. Significant bleeding was rarely seen, with the lowest incidence in IAS alone. A recently published case series also showed that IAS better preserved fibrinogen concentrations as compared to TPE [12]. These findings may have clinical implications. In fact, TPE might be supplanted by IAS as the latter technique appears to be safer with regard to fibrinogen handling. In addition, IAS allows almost full clearance of a vast majority of circulating Ig (sub)types without the need for concomitant FFP or albumin substitution. This might explain successful rescue treatment with IAS in some 'TPE-resistant' cases [16]. Though not yet well-established, treatment generally consists of two IAS sessions, executed within the first $48 \mathrm{~h}$, to obtain a rapid decrease in Ig levels followed by repeated treatment cycles to overcome the redistribution of pathological antibodies [17].

In conclusion, the study of Zöllner et al. is original and challenging but must be interpreted with caution since results were obtained retrospectively in a small and heterogeneous patient population. The observed advantages (low bleeding risk and more optimal Ig clearance) and cost-effectiveness of IAS as compared with TPE need confirmation by large prospective randomized trials $[18,19]$.

\section{KARGER}

(C) 2014 S. Karger AG, Basel

0253-5068/14/0382-0158\$39.50/0
Prof. Patrick M. Honoré, MD, PhD, FCCM

ICU Department, UZ Brussel - VUB University

101, Laarbeeklaan

BE-1090 Jette, Brussels (Belgium)

E-Mail Patrick.Honore@ uzbrussel.be 


\section{References}

1 Hildebrand AM, Huang SH, Clark WF: Plasma exchange for kidney disease: what is the best evidence? Adv Chronic Kidney Dis 2014; 21:217-227.

-2 Ramírez-Bueno A, Salazar-Ramírez C, CotaDelgado F, de la Torre-Prados MV, Valdivielso P: Plasmapheresis as treatment for hyperlipidemic pancreatitis. Eur J Intern Med 2014; 25:160-163.

-3 Hohenstein B, Bornstein SR, Aringer M: Immunoadsorption for connective tissue disease. Atheroscler Suppl 2013;14:185-189.

4 Brunskill SJ, Tusold A, Benjamin S, Stanworth SJ, Murphy MF: A systematic review of randomized controlled trials for plasma exchange in the treatment of thrombotic thrombocytopenic purpura. Transfus Med 2007;17:17-35.

5 Orlin JB, Berkman EM: Partial plasma exchange using albumin replacement: removal and recovery of normal plasma constituents. Blood 1980;56:1055-1059.

6 Szodoray P, Hajas A, Toth L, Szakall S, Nakken B, Soltesz P, Bodolay E: The beneficial effect of plasmapheresis in mixed connective tissue disease with coexisting antiphospholipid syndrome. Lupus 2014;23:1079-1084.

$>7$ Berlot G, Tomasini A, Silvestri L, Gullo A: Plasmapheresis in the critically ill patient. Kidney Int Suppl 1998;66:S178-S181.
-8 O’Brien KL, Price TH, Howell C, Delaney M: The use of $50 \%$ albumin/plasma replacement fluid in therapeutic plasma exchange for thrombotic thrombocytopenic purpura. Clin Apher 2013;28:416-421.

$>9$ Yeh JH, Chiu HC: Coagulation abnormalities in serial double-filtration plasmapheresis. J Clin Apher 2001;16:139-142.

10 Shibata Y, Shigemi D, Ito M, Terada K, Nakanishi K, Kato M, Igarashi M, Suzuki S: Association between fibrinogen levels and severity of postpartum hemorrhage in singleton vaginal deliveries at a Japanese perinatal center. J Nippon Med Sch 2014;81:94-96.

11 Bonello VA, Bhangu A, Fitzgerald JE, Rasheed S, Tekkis P: Intraoperative bleeding and haemostasis during pelvic surgery for locally advanced or recurrent rectal cancer: a prospective evaluation. Tech Coloproctol 2014; 18:887-893.

-12 Koessler J, Kobsar A, Kuhn S, Koessler A, Yilmaz P, Weinig E, Putz E, Boeck M, Klinker E: The effect of immunoadsorption with the Immusorba TR-350 column on coagulation compared to plasma exchange. Vox Sang 2014, DOI: 10.1111/vox.12191, Epub ahead of print.
13 McLeod BC: Plasma and plasma derivatives in therapeutic plasmapheresis. Transfusion 2012;52(suppl 1):38S-44S.

14 Klingel R, Heibges A, Fassbender C: Plasma exchange and immunoadsorption for autoimmune neurologic diseases - current guidelines and future perspectives. Atheroscler Suppl 2009;10:129-132.

15 Zöllner S, Pablik E, Druml W, Derfler K, Rees A, Biesenbach P: Fibrinogen reduction and bleeding complications in plasma exchange, immunoadsorption and combination of the two. Blood Purif 2014;38:160-166.

16 Waters AM, Licht C: aHUS caused by complement dysregulation: new therapies on the horizon. Pediatr Nephrol 2011;26:41-57.

17 Rummler S, Barz D: Plasma exchange and immunoadsorption of patients with thoracic organ transplantation. Transfus Med Hemother 2012;39:234-240.

18 Seck SM, Bertrand D, Boucar D: Current indication of plasma exchanges in nephrology: a systematic review. Saudi J Kidney Dis Transpl 2011;22:219-224.

19 Casian A, Jayne D: Plasma exchange in the treatment of Wegener's granulomatosis, $\mathrm{mi}$ croscopic polyangiitis, Churg-Strauss syndrome and renal limited vasculitis. Curr Opin Rheumatol 2011;23:12-17. 